\title{
MULTI-OBJECT VIDEO RATE CONTROL
}

\author{
Nadjib Achir, Guy Pujolle \\ University of Paris 6, LIP6 Lab. 8 rue du Capitaine Scott 7501, Paris Nadjib.Achir@lip6.fr. \\ Guy.Pujolle@lip6.fr
}

\begin{abstract}
The ISO MPEG-4 standard enhances the traditional concept of video sequence and proposes a synchronized set of visual objects organized in a flexible way. The video delivery according to this new paradigm results in a challenging new bit-allocation, and rate-control mechanisms, which has to satisfy complex application requirements. In this paper, we propose a rate control mechanism, which optimally selects the sending rate for each object constituting the video scene. This rate allocation is done in agreement with the particularity of the multi-object video coding schemes. Thus, in our proposal, we allocate more bandwidth to the object having the most impact on the video quality. To perform this optimal allocation, we use the dynamic programming approach to select the optimal amount of bandwidth to be allocated to each object. The obtained results show the importance of this consideration in multi-object video delivery. We demonstrate that the video quality can be significantly increased.
\end{abstract}

\section{INTRODUCTION}

One of the greatest challenges of the past years has been the delivery of video streams over packet networks, with the best effort service model. In such environment, multimedia terminals are confronted with fluctuating packet loss rates and bandwidth. Thus, video source must be able to adapt video flow rates to the available bandwidth in order to guarantee the best possible quality reconstructed video at the decoder. Another consequence of the increasing role of video is an important evolution in the concept of audiovisual information. While for a very long time video processing dealt exclusively with fixed-rate sequences of rectangularshaped images, interest is recently moving toward a more flexible concept. The subject of the processing and encoding operations, in this case, is a set of visual elements/objects (Video Object) organized in both time and space in a flexible and arbitrary complex way. The ISO MPEG-4 [1] international standardization effort supports this new advanced concept of visual information for covering the generic

The original version of this chapter was revised: The copyright line was incorrect. This has been corrected. The Erratum to this chapter is available at DOI: 10.1007/978-0-387-35703-4_21

D. Gaïti et al. (eds.), Network Control and Engineering for QoS, Security and Mobility II

(C) IFIP International Federation for Information Processing 2003 
coding of audiovisual information for a wide range of rates and applications. Thus, using MPEG-4 standard, it is possible to encode the video scene into a separate video object and also transmit a compositing script that describe how these video object need to be composed to make a compelling presentation at the receiver's decoder. One of the advantages of this approach is that, depending on the channel bandwidth and the relative importance of each video object in the video scene, it is possible to control undependably the different video object. Thereby, we can achieve more efficient utilization of the available outgoing bandwidth. For example, if we consider a video-conference system with speaker and background as different objects. The only interest for users is to obtain the best possible quality for the most relevant object (i.e. the speaker). We can then, allocate more bandwidth to the video object representing the speaker than to the background. Thus the receiver perceptual video quality can significantly enhance.

The main contribution of this work is the proposition of an optimized bandwidth distribution among the different video's object constituting the video scene, in order to enhance the video quality metric at the receiver. This video quality metrics represent the user satisfaction and depend on the quality and the priority of each object in the video scene. We model the per object video quality by computing the distortion introduced by each object on the received video. Our proposed formulation is simple and allows us to show how we can optimally allocate the global outgoing bandwidth budget between the different objects. The obtained results show the importance of this consideration in the multi-object video delivery, and demonstrate that the video quality can be significantly increased.

The rest of the paper is organized as follows: in Section 2, we model the multiobject video quality using a rate distortion function. In Section 3 , we present the proposed architecture. In this section we formalize the object-based rate distortion optimization problem, namely, the assigning of more bandwidth budget to the more important video objects, in order to maximize the received video quality. Then, to solve this optimization problem, we use the dynamic programming approach. Our simulation results, presented in Section 4, demonstrate that the perceptual video quality at the decoder can be significantly reduced. Finally, we present the conclusions in Section 5.

\section{MPEG-4: OVERVIEW}

While MPEG-1 and MPEG-2 video compression techniques are restricted to rectangular pictures from natural video, the scope of MPEG-4 visual is much wider. MPEG-4 defines a framework for joint description, compression, storage, and transmission of natural and synthetic audiovisual information. It defines improved compression algorithms for audio and video signals, including specialized tools, (e.g., speech coding). The visual information is organized on the basis of the visual object concept, which represents a time-varying visual entity with arbitrary shape. This visual object can be individually manipulated and combined with other similar entities to produce a scene. The MPEG-4 standard includes very efficient compression techniques. 
MPEG-4 encoded data stream can be described in an hierarchical manner as shows Fig.1. The highest syntactic structure is the visual object scene. It consists of one or more visual objects. Each visual object belongs to one of the following object types: video object, still texture object, mesh object, and face object. In the following, we will detail only video objects (VO).

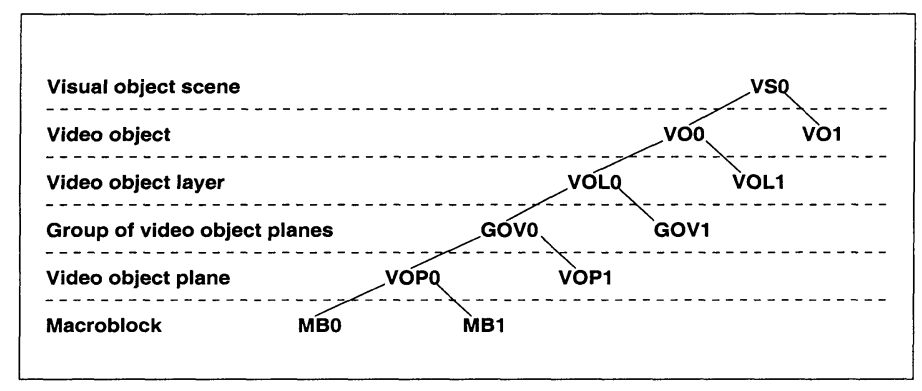

Figure 1: Syntactic structure for video object of an MPEG-4 visual encoded data stream.

Natural video objects (VO) are encoded in one or more video object layers $(V O L)$-- One base layer and one or several enhancement layers. Each layer includes information corresponding to a given level of temporal or spatial resolution, so that scalable transmission and storage are allowed. In single layer coding, only one video object layer exists. Each VOL contains a sequence of 2D representations of arbitrary shapes at different time intervals that is referred to as a video object plane (VOP). These VOPs can be structured in groups of video object planes $(G O V)$. VOPs can be coded (as in MPEG-1 and MPEG-2 standards) as an I-VOP (without temporal prediction), $P$-VOP (with prediction from the previous $I-V O P$ ), or $B$-VOP (with predictions from a previous and future $V O P s$ ). Our work considers objects that are represented by a single $V O P$.

\section{MULTI-OBJECT VIDEO RATE CONTROL}

We have shown above that the multi-object video coding opens some new perspectives in video delivery over Internet. Since the scene is subdivided into different objects with different priorities, the source coder have some indications to compute the rate of each object according to there scene priority and provide the better video quality at the receiver side. Thus, our approach is to determine, at the source, the portions of the outgoing bandwidth to allocate to each object. In Fig.2 we illustrate the architecture of a multi-object video rate control system. The system architecture comprises two main components: a target bandwidth computation module and a Multi-Object video bandwidth allocation module. The target bandwidth computation module estimates the available bandwidth between the source and the receiver. And, the Multi-Object video bandwidth allocation module optimally distributes this estimated bandwidth among the different object of the scene. This allocation is done according to the priority and the perceptual quality of 
the video objects in the scene. In our works, we represent this perceptual quality by the distortion introduced by each video object in the video. In the following, we will describe each module and we will give a cost function representing the perceptual quality of the video according to the rate and the priority of each object.

\subsection{Target Bandwidth Computation}

We now consider the problem of designing a flow control algorithm for the video communication. This flow control algorithm will give us the available bandwidth between the source and the receiver. Actually, the Internet stability depends principally on the end-to-end congestion control. The applications which do not perform congestion control will be hardly penalized by the network, like video or audio applications which employ non-TCP protocols to transmit continuous media. To achieve fair sharing of networking bandwidth, it is advantageous that non-TCP protocol share resources fairly with others instances of itself and with TCP, the dominant source of Internet traffic. TCP cannot be used directly for video transport because its reliability is achieved at the expense of time varying delay and throughput. In recent years, several researches have been devoted into investigation on the TCP-friendly rate control. The TCP-Friendly Rate Control Protocol (TFRC) [3], is a rate-based end-to-end congestion control protocol. TFRC uses a model for steady state TCP throughput to limit the sending rate and assure fair behavior against competing flows. Instead of reacting to single congestion events (in the form of packet loss) like TCP, the TFRC protocol changes its sending rate in response to the loss rate, sampled over a certain amount of time. While TFRC achieves the same long-term throughput as a conformant TCP flow, its short-term sending rate is more stable. This makes the protocol suitable for traffic where sudden rate changes are undesirable as video and audio delivery. In our work we consider, that the video source implements the TFRC protocol to regulate the video traffic.

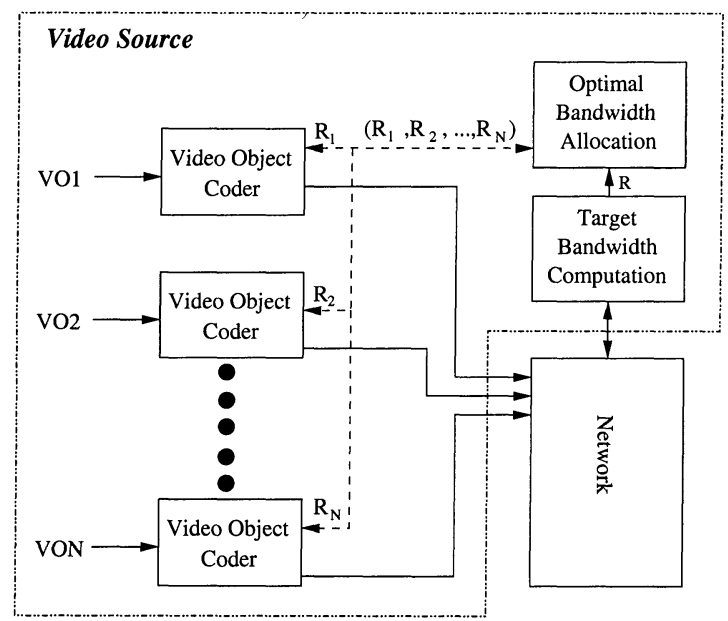

Figure 2 : Multiobject video rate control system. 


\subsubsection{Multi-Object Video Quality Modeling}

The best and generic way to translate user and application requirements in to control objectives is the definition of a cost function whose value has to be minimized or maximized by the control system. In this work, we consider a cost function called Weighted Distortion introduced in [2]. The scope of this cost function is the minimization of the weighted average of the distortion of the different objects. The distortion is defined as the mean squared value of the difference of the pixels of original and decoded pictures, usually termed mean squared error (MSE). In the following, we assume that our video source is able to generate a multi-object based video coding. This video is composed of a set of objects $\mathrm{O}=\left\{O_{i}\right\}_{i \in\{1, N\}}$. In this case, the cost function can be denoted as following

$$
D_{\text {scene }}=\sum_{i=1}^{N} \alpha_{i} \times D_{i}
$$

where $D_{\text {scene }}$ correspond to the global distortion of the video scene, $D_{i}$ corresponds to the distortion of the object $O_{i}$, and $\alpha_{i}$ are weighting factors corresponding to the object priority in the scene $\left(\sum_{i=1}^{N} \alpha_{i}=1\right)$. Object priority can either be indicated by users or be based on heuristics that take into account content's features like object's position, speed, and complexity. Thus, by assigning larger factors to the more important objects, the encoder is indirectly informed to be more careful with them than with other less important objects. To represent the distortion, several studies lead to the conclusion that the distortion evolves somehow exponentially with the decreasing source rate [4] [5]. Therefore, the per object perceptual distortion-rate function, at the source, can be expressed as

$$
D_{i}\left(R_{i}^{(s)}\right)=D_{i}^{\max } \times 2^{-\beta_{i} R_{i}^{(s)}} .
$$

The parameter $\beta_{i}$ is related to the encoding complexity of the object $O_{i}$, and $R_{i}^{(s)}$ denotes the maximum rate of the object at the source. In this rate/distortion model, it will be assumed that the distortion is upper bounded by some value $D_{i}^{\max }$. We suppose that $D_{i}^{\max }=1$. Fig.4 represents the perceptual video distortion evolution with different video rate values. We observe that the distortion varies exponentially. A small increase in the source rate leads to a great decrease in perceptual distortion. 


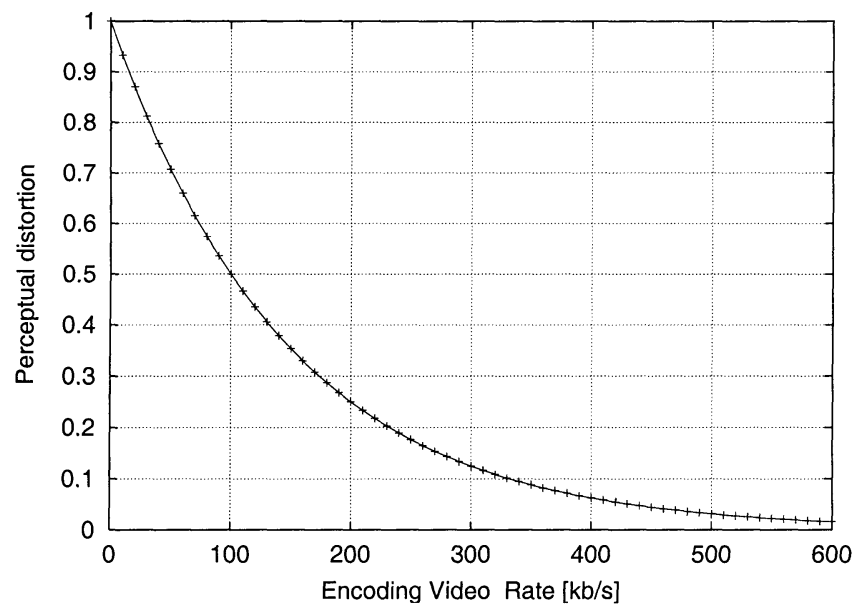

Figure 3: Perceptual distortion as a function of the video rate. For $\beta_{i}=0.01$, and $\mathrm{R}=600 \mathrm{~kb} / \mathrm{s}$

The cost function represented by (1), represent the user quality satisfaction at the source. But, at the receiver, this quality may change principally due to the packet losses introduced by the network. Thus, to determine the cost function at the receiver side we must take into consideration the network packet loss rate. In our paper, we assume that each object $O_{i}$ is in the form of a succession of VOP, completely independent one of the others. Therefore, if one VOP is lost or incompletely received, they it does not affect any of the following VOP. Each $V O P_{i}$, belonging to an object $O_{i}$, is subdivided into $P_{i}$ packets. We assume in the following, that the Packet Error Rate probability induced by the network for each packet is equal to $\varepsilon_{P E R}$. In this case, the Frame Error Rate, $\varepsilon_{F E R}$, can then be denoted as

$$
\varepsilon_{F E R}=1-\left(1-\varepsilon_{P E R}\right)^{P_{i}} .
$$

If all the $P_{i}$ packets of $V O P_{i}$ are correctly received at the decoder, thereby all the $V O P_{i}$ can be correctly decoded with a distortion equal to

$$
D_{i}=\left(1-\varepsilon_{F E R}\right) \times D_{i}^{\max } \times 2^{-\beta_{i} R_{i}^{(r)}} .
$$

On the other hand, if one of the $P_{i}$ packets are not received correctly, then we assume that the distortion perceived at the receiver for $O_{i}$ is equal to $D_{i}^{\max }$. This assumption is some what pessimistic, but in practices the loss of part of the description of the VOP is not equivalent to a complete loss of the frame encoding. From (3) and (4) we can compute, for each object $O_{i}$, the observed distortion at the receiver. This distortion depend on the allocated rate $R_{i}$ for $O_{i}$, as indicated in the following 


$$
D_{i}\left(R_{i}\right)=\left\lfloor\varepsilon_{F E R} \times D_{i}^{\max }+\left(1-\varepsilon_{F E R}\right) \times D_{i}^{\max } \times 2^{-\beta_{i} R_{i}^{(r)}}\right\rfloor
$$

The global-video distortion can then be denoted as the sum of all the $D_{i}\left(R_{i}\right)$ multiplied by the associated priority, $\alpha_{i}$ for $i=1, \ldots, N$, as indicated by the following equations:

$$
D_{i}(R)=\sum_{i=1}^{N} \alpha_{i} \times\left[\varepsilon_{F E R} \times D_{i}^{\max }+\left(1-\varepsilon_{F E R}\right) \times D_{i}^{\max } \times 2^{-\beta_{i} R_{i}^{(r)}}\right]
$$

where $R$ is the global bandwidth budget available at the source (i.e. $R=\sum_{i=1}^{N} R_{i}$ ). To guaranties the best quality at the receiver side we must then minimize the equation (6) with respect to the $R_{i}$ 's.

\subsection{Multi-Object Video Bandwidth Allocation}

In the last section we shown that, to enhance the video quality at the receiver side in the case of unicast delivery, we must minimize (6) with respect to the $R_{i}$ 's. This optimization is done by optimally distribute the available bandwidth among the video objects. To solve this problem we will use, in our paper, a mathematical optimization method, called dynamic programming. The Dynamic Programming [6] is an optimization procedure that is particularly applicable to discrete-time dynamic system, requiring a sequence of interrelated decisions. Each decision transforms the current system state into a new state. Such sequence of decisions, which in turn yields a sequence of situations, maximizes (or minimizes) some cost function. The basic model of the dynamic programming has two principal features: $(i)$ an underlying discrete-time dynamic system, and (ii) a cost function that is additive over time. The dynamic system is on the form

$$
x_{k+1}=f_{k}\left(x_{k}, u_{k}\right), \quad k=0,1, \ldots, N-1,
$$

where $k$ is an indexes discrete time, $x_{k}$ is the state of the system and summarizes past information that is relevant for future optimization. $x_{k}$ is an element of a space $S_{k} . u_{k}$ is the control or decision variable to be selected at time $k . u_{k}$ is an element of space $C_{k}$. The control $u_{k}$ is constrained to take values is a given nonempty subset $U\left(x_{k}\right) \subset C_{k}$, which depends on the current state $x_{k}$; that is, $u_{k} \in U\left(x_{k}\right)$ for all $x_{k} \in S_{k}$ and $k$. And finally, $N$ is the horizon or number of time control is applied. The sequence $\pi=\left\{u_{0}, u_{1}, \ldots, u_{N-1}\right\}$, which is the set of control decisions over the time control, will be referred as an admissible policy. The cost function is additive in the sense that the cost incurred at time $k$, denoted $g_{k}\left(x_{k}, u_{k}\right)$, accumulates over time. Thus, for each $\pi$, the corresponding expected cost for a fixed initial state $x_{0}$ is 


$$
J_{\pi}\left(x_{0}\right)=\underset{k=0,1, \ldots, N-1}{E}\left[g_{N}\left(x_{N}\right)+\sum_{k=0}^{N-1} g_{k}\left(x_{k}, u_{k}\right)\right] .
$$

Then the optimal policy $\pi^{*}$ is one that minimizes this cost; that is,

$$
J_{\pi^{*}}\left(x_{0}\right)=\min _{\pi \in \Pi} J_{\pi}\left(x_{0}\right),
$$

where $\Pi$ is the set of all admissible policies. The optimal cost $J_{\pi^{*}}\left(x_{0}\right)$ of this basic problem is equal to $J_{0}\left(x_{0}\right)$, where the function $J_{0}$ is given by the last step of the following algorithm:

$$
\left\{\begin{array}{c}
J_{N}\left(x_{N}\right)=g_{N}\left(x_{N}\right), \\
J_{k}\left(x_{k}\right)=\min _{\pi \in \Pi} E\left[g_{k}\left(x_{k}, u_{k}\right)+J_{k+1}\left(f\left(x_{k}, u_{k}\right)\right)\right],
\end{array}\right.
$$

which proceeds backward in time from the period $N-1$ to period 0 .

To apply this optimization method to our problems we will have to define: our system function, the steps of our system, and finally a cost function. In our system, we consider that the step number is equal to the number of object constituting the scene $(N)$. Thus, the $k^{\text {th }}$ step corresponds to the $k^{\text {th }}$ object $\left(O_{k}\right)$. We consider also that the decision variable, $u_{k}$, to be selected at step $k$, corresponds to the amount of bandwidth allocated to the object $O_{k}$. The state of the system, $x_{k}$, corresponds to the amount of bandwidth available for the objects $\{(k),(k+1), \ldots,(N)\}$. In this case, the global bandwidth, $R$, available for all the video is equal to the initial state $x_{1}$. We seen above that decision variable $u_{k}$ take values in $U\left(x_{k}\right)$. Thus, to obtain a finite state number per step, we must discrtize the set $U\left(x_{k}\right)$. To achieve this discretisation of $U\left(x_{k}\right)$, we divide the among of bandwidth available into $M$ pieces using the discretization interval $l=\frac{R}{M}$. In the following, $M$ is referred as the discretisation index. Under those assumptions our discrete-time dynamic system can be formulated by

$$
x_{k+1}=f_{k}\left(x_{k}, u_{k}\right)=x_{k}-u_{k}, \quad k=1,2, \ldots, N,
$$

This means that the amount of bandwidth available for the objects $\{(k+1),(k+2), \ldots,(N)\}$, is equal to the amount of bandwidth available for the objects $\{(k),(k+1), \ldots,(N)\}$, minus the bandwidth allocated to the object $O_{k}$. In this case, the cost function of the $k^{\text {th }}$ step is represented by the distortion introduced by the $k^{\text {th }}$ object (5) multiplied by the priority of this object $\left(\alpha_{k}\right)$.

$$
g_{k}\left(x_{k}, u_{k}\right)=\alpha_{k} \times D_{k}\left(u_{k}\right) \text {. }
$$


This cost function has the particularity to be additive in time, since the global distortion of the video scene is equal to the sum of the distortion of each individual object multiplied by their priority.

With these notations, the Dynamic-Programming algorithm can be described with an initial state equal to

$$
J_{k}\left(x_{k}\right)=g_{N}\left(x_{N}\right)=\alpha_{N} \times D_{N}\left(u_{N}\right) .
$$

which can be easily computed for all possible $u_{k} \in U\left(x_{k}\right)$. And by a cost-togo function $J_{k}\left(x_{k}\right)$ defined as:

$$
J_{k}\left(x_{k}\right)=\min _{\pi \in \Pi} E\left[\alpha_{k} \times D_{k}\left(u_{k}\right)+J_{k+1}\left(x_{k}-u_{k}\right)\right],
$$

where $\pi=\left\{u_{1}, u_{2}, \ldots, u_{N-1}\right\}$.

This function $J_{k}\left(x_{k}\right)$ denotes the optimal expected cost for the remaining periods when starting at period $k$ and with initial available bandwidth budget $x_{k}$. The function $J_{k}\left(x_{k}\right)$ is computed recursively backward in time, starting at period $N-1$ and ending at period 1 . The value $J_{1}(R)$ is the optimal expected cost for the process when initial bandwidth at time $k=1$ is $R$. During the computation, the optimal policy $\pi^{*}=\left\{u_{1}^{*}, u_{2}^{*}, \ldots, u_{N-1}^{*}\right\}$, which represent the optimal bandwidth allocation, is simultaneously computed from the minimization in the right-hand of (14). Thus, at the end of the algorithm we obtain the optimal distribution of the bandwidth $R$ among the $N$ object constituting the video scene. This optimal distribution $R^{*}$ is equal to:

$$
R^{*}=\left(u_{1}^{*}, u_{2}^{*}, \ldots, u_{N-1}^{*}\right)
$$

\section{PERFORMANCES ANALYSIS}

In this section we present the obtained results for our analysis. We concentrate our study on a single source $S$ and a single receiver $R$. We model the outgoing source channel as having independent packet loss rate. We assume, in the following, that the source $S$ generates a multi-object based video coding, and the receiver $R$ is able to compose the video scene from the received objects. We suppose, that the video scene is composed of three objects $O_{1}, O_{2}$, and $O_{3}$. The weighted average of the three objects is equal to $0.5,0.3$, and 0.2 respectively. Thus the most important object is $O_{1}$ and the less important one is $O_{3}$. The maximum video source rate, for each object, is equal to $600 \mathrm{kbps}$ for $\mathrm{O}_{1}, 400 \mathrm{kbps}$ for $\mathrm{O}_{2}$, and 200 kbps for $\mathrm{O}_{3}$. The source $S$ implement the TFRC protocol introduced in the subsection 3.1, in order to compute the available bandwidth between $S$ and $R$. In our analysis, we distribute the bandwidth among the video objects following two 
approaches: The first one is the dynamic programming approach proposed in this paper, and the second one is the Naïf approach. The Naïf approach is in the form of a fairly bandwidth allocation between the different objects constituting the video scene. In this case, the priorities between the objects are not taken into consideration. All the objects are considered important as indicated by the following algorithm:

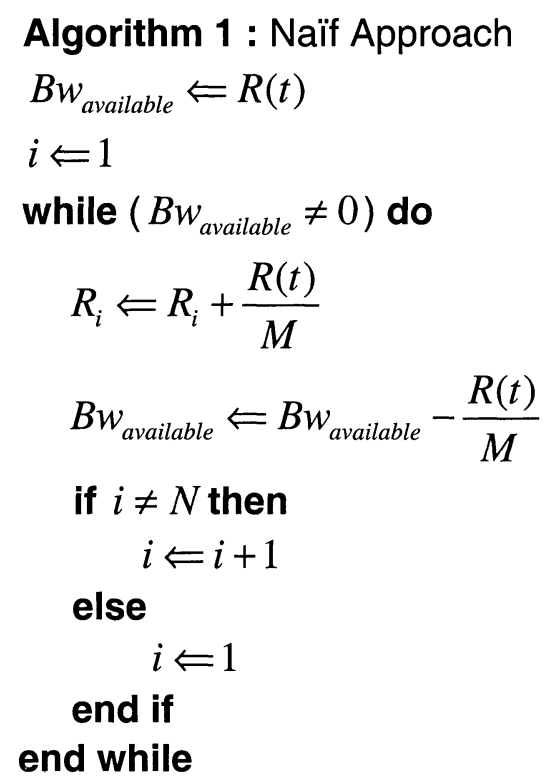

In Fig.4, we shows the perceptual distortion performance obtained at the receiver for each of the bandwidth allocation approach proposed above (i.e. the Dynamic Programming approach, and the Naif approach). The distortion axis represents the average of the multi-object's video distortion defined in (6). We suppose that the packet error rate in the network is equal to $5 \%$. We vary the available bandwidth on the outgoing source link and we compute, for each bandwidth value, the corresponding distortion perceived at the destination. As the figure demonstrates, our result compares favorably with the Naif approach. This is justified by the fact that the dynamic programming based allocation draws part of the priorities between the objects. It allocates more bandwidth to the object having the most impact on the perceptual video quality.

To investigate the impact of the object priority on the user satisfaction quality, we use a fairness index [7]. This fairness index, $F$, is defined to be a function between 0 and $1 . F$ is equal to 1 , if all the priorities of the objects are identical, and is close to 0 if a large fraction of the sum of the priorities is concentrated in one priority. This fairness function is denoted as 


$$
F=1-\frac{1}{2 \times(N-1)} \sum_{i=1}^{N}\left|\frac{\alpha_{i}}{\mu}-1\right|,
$$

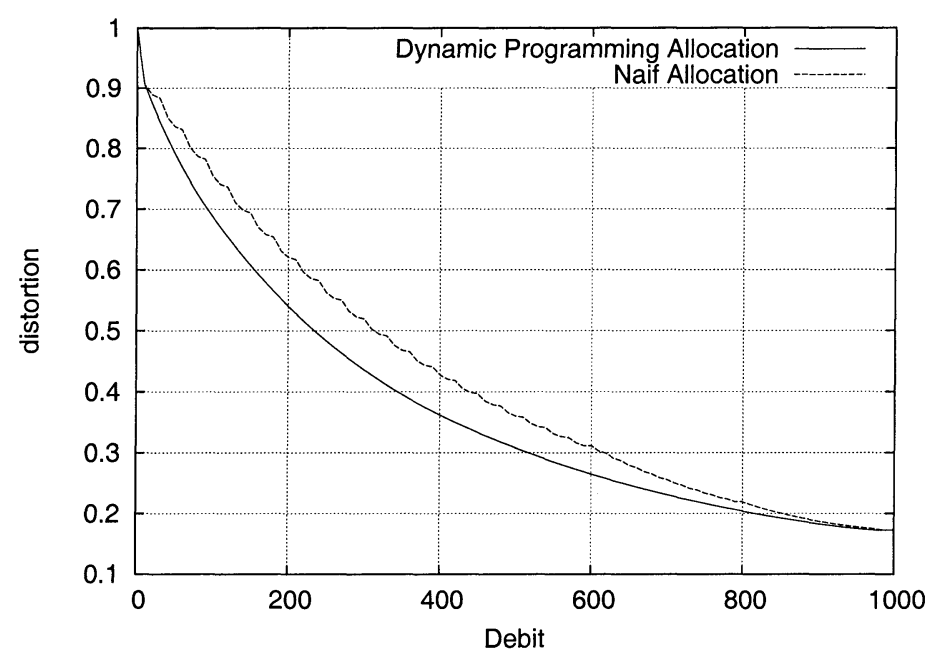

Figure 4: Perceptual distortion of the video at the receiver.

Table 1:Objects priority.

\begin{tabular}{|l|l|l|l|}
\hline Priority $O_{1}\left(\alpha_{1}\right)$ & Priority $O_{2}\left(\alpha_{1}\right)$ & Priority $O_{1}\left(\alpha_{1}\right)$ & Fairness $(\mathrm{F})$ \\
\hline 0.334 & 0.333 & 0.333 & 0.999 \\
\hline 0.4 & 0.3 & 0.3 & 0.9 \\
\hline 0.4 & 0.4 & 0.2 & 0.8 \\
\hline 0.53 & 0.3 & 0.17 & 0.705 \\
\hline 0.6 & 0.3 & 0.1 & 0.6 \\
\hline 0.665 & 0.22 & 0.115 & 0.5025 \\
\hline 0.73 & 0.22 & 0.05 & 0.405 \\
\hline 0.8 & 0.15 & 0.05 & 0.3 \\
\hline 0.865 & 0.12 & 0.015 & 0.2025 \\
\hline $\mathbf{0 . 9 3}$ & $\mathbf{0 . 0 6}$ & $\mathbf{0 . 0 1}$ & 0105 \\
\hline
\end{tabular}

$\mu$ is the average priority. $\alpha_{i}$ denote the object priority and $N$ the number of object in the video scene. In Table.1 we summarize the per object priority values, and their respective fairness, chosen for our analysis. We compute the distortion, perceived at the receiver, for the different values of fairness indicated in this table. We plot, in Fig.5, the gain obtained for each values of $F$ with several values of the network available bandwidth. This gain represents the difference between the distortion obtained in the case of the Naif approach allocation and dynamic programming approach allocation. We can observe that, the gain increase as the fairness index increase. If the video objects have equivalent priorities $(F<0.2)$, then dynamic programming approach and naive allocation approach give the same 
result. On the other hand, if the video scene comprises a very important objects. Then, a great gain can be obtained. Our proposal is very powerful for video applications that contain objects with different impact on the video, as the video conferencing system.

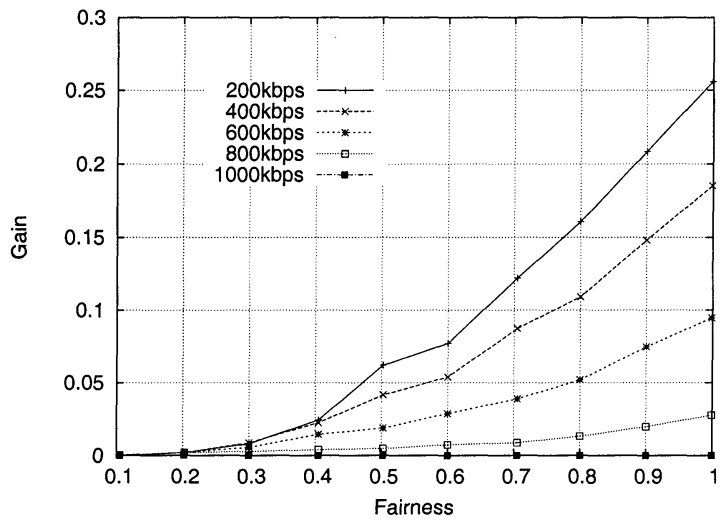

Figure 5: Video object fairness Vs Available Bandwidth

\section{CONCLUSION}

We have shown in this paper that object-based coding of video sequences enables exploration of new type of media scaling. For this reason we propose an optimized distribution of the available bandwidth among the different video objects, to achieve more efficient utilization of the outgoing bandwidth. To ensure this optimal allocation of the bandwidth between the objects we use the dynamic programming approach. This allocation is done according to the priority and the rate/distortion function of each object of the video. The obtained results show that the video quality at the receiver can be significantly enhanced.

\section{REFERENCES}

[1] ISO/IEC 14496-1, "Information Technology - Coding of Audio-visual Objects, Part 1 : System," in ISO/IECJT1/SC 29/WG 11 Draft International Standard, Dec, 1998.

[2] J. I. Ronda, M. Eckert, "Rate Control and Bit Allocation for MPEG-4," in Proc. of IEEE Transactions on Circuits and Systems for Video Technology, Dec. 1999.

[3] S. Floyd, M. Handley, J. Padhye, and J. Widmer, " Equation-based congestion control for unicast applications", in SIGCOMM 2000, Aug, 2000.

[4] J. Bai, Q. Liao, X. Lin, "Hybrid models of the rate distortion characteristics for MPEG video coding," in 7th International Conference on Communication Technology, Aug, 2000.

[5] J. Lin, A. Ortega, "Bit-Rate Control Using Piecewise Approximated Rate-Distortion Characteristics,"in Proc. of IEEE Transactions on Circuits and Systems for Video Technology, Aug. 1998.

[6] R. E. Bellman, "Dynamic Programming," in Princeton Univ. Press, Princeton, N.J., 1957.

[7] L. Vicisano, L. Rizzo, and J. crowcroft, " TCP-like congestion control for layered multicast data transfer", in Proc. of IEEE Infocom, Mar. 1998. 